\title{
Leaders on a Mindfulness-Based Program: Experience, Impact, and Effect on Leadership Role
}

\author{
Deryl Dix ${ }^{1} \cdot$ Katie Norton ${ }^{1} \cdot$ Gemma M. Griffith $^{1}$
}

Accepted: 4 January 2021 /

Published online: 14 January 2021

(c) Crown 2021

\begin{abstract}
Although there is putative evidence that mindfulness-based programs (MBPs) may contribute to leadership skills, little is known about the direct experience of leaders who attend such programs. It is therefore unknown how MBPs delivered in the corporate environment are experienced by leaders, or how MBPs may facilitate leadership development. This qualitative study explored how leaders experienced an MBP, introduced as a part of a wider leadership development program, and the impact of this intervention on their work lives and leadership role. Participants $(N=10)$ were leaders who worked for a global manufacturing organization and who had attended a three-day mindfulness-based program as part of a leadership development program. They were interviewed and the data was analysed using thematic analysis. Following the MBP, eight participants reported enhanced emotional awareness, and a greater understanding of the impact of work-related stress, which in turn helped their leadership role. Two did not see how mindfulness could enhance their leadership skills although the MBP was well received by most of the participants. Three superordinate themes were identified: (1) The participant journey: Mixed experiences of the MBP; (2) A clash of cultures: The challenge of integrating mindfulness into the workplace, and (3) Impact on leadership. These results may inform future research, program design, and implementation.
\end{abstract}

Keywords Leadership · Mindfulness · Stress · Qualitative · Management · Workplace

Mindfulness is defined as deliberately paying attention to present moment experience, in a non-judgmental way (Kabat-Zinn, 2013). Mindfulness is a complex construct to define and has been used by researchers to "describe a theoretical construct (mindfulness), a practice of cultivating mindfulness (such as meditation), or a psychological process (being mindful)" (Germer, 2005, p. 6). Since Jon Kabat-Zinn pioneered Mindfulness-Based Stress Reduction (MBSR) courses in the late 1970s, other forms of mindfulness-based programmes (MBPs)

Gemma M. Griffith

g.m.griffith@bangor.ac.uk

Deryl Dix

d.dix@ntlworld.com

1 Centre for Mindfulness Research and Practice (CMRP), School of Psychology, Bangor University, Wales, UK 
for particular populations have been developed, including Mindfulness-Based Cognitive Therapy (MBCT) for people with persistent depression (Segal et al., 2013). The research available from clinical settings report participant reductions in anxiety, depression, and stress following an MBP (Baer, 2003; Kuyken et al., 2008,2015). There may also be other benefits beyond reducing negative affect, including building positive qualities such as selfawareness and compassion (Khoury et al., 2015). More recently, researchers have focused on non-clinical populations, such as people in the workplace (Hyland et al., 2015; King \& Haar, 2017; Reitz et al., 2016). In particular, mindfulness as a tool to support leadership has attracted popular and academic interest (Chaskalson, 2011; Hyland et al., 2015).

Enquiries following the economic crash in 2008 pointed to the failure of governance and leadership (The Financial Services Authority Board, 2011). Since then, there have been calls for authentic leaders with high levels of emotional intelligence (Northouse, 2013), with several authors proposing that self-awareness is an essential component of the authentic style of leadership (Hughes et al., 2009; Northouse, 2013). Additionally, many organizations have developed models which "distinguish leadership as a way of being and focus on the interpersonal development of the self as a leader' (Brendel \& Bennett, 2016, p. 410). This may explain some of the recent interest among businesses and researchers in embedding MBPs within leadership development programs.

The meaning of leadership is a complex and hotly debated area. Some definitions focus on the relationship between leaders and their team members, others on the process of leadership, some on leadership competencies, and yet others on personality (Gill, 2011; Hughes et al., 2009). Several authors who advocate mindfulness-based leadership development have discussed the challenges of leadership in the current highly pressured, complex global environment (Brendel et al., 2016; Reitz et al., 2016). The acronym "PAID" was created to describe how leaders within this environment can experience feeling "Pressured, Always-on, Information Overloaded, and Distracted" (Hougaard et al., 2016, p. 49). These authors note that leaders can become "victims" of this culture, resulting in a detrimental impact for them and their organizations.

Hougaard et al. (2016) described mindfulness as a potential tool which could provide a foundation for leadership, and Ehrlich (2017) wrote, "When we actively pay attention, we build self-awareness, which is the heart of leadership" (p. 234). Many organizations have implemented MBPs for leaders (Brendel \& Bennett, 2016; Mindfulness All Party Parliamentary Group, 2015), and there is emergent evidence that trait mindfulness in leaders has a positive impact on dimensions of leadership (King \& Haar, 2017) and that leaders who have attended an MBP report lower stress and greater well-being (Reb et al., 2014; Wasylkiw et al., 2015). Several models of "mindful leadership" have emerged, which propose that MBPs may support leaders to operate more effectively (Ehrlich, 2017; Hougaard et al., 2016; Reitz et al., 2016), but little is known about what it is specifically about MBPs that may facilitate these reported changes.

As is common in MBP research, most studies on leadership have focused on stages I and II of the National Institutes of Health (NIH) stage model (Dimidjian \& Segal, 2015; Onken et al., 2014) concerning intervention generation/refinement, and efficacy of interventions. As many organizations are already offering MBPs for workers and leaders, it is important to balance the development research with research on how these are implemented and experienced by participants (i.e., NIH Stages IV and V). Dimidjian and Segal (2015) identified the dearth of Stage IV and V studies in MBP research, with under $2 \%$ of the MBP studies they reviewed falling into these two categories. They called for more research based on implementation and dissemination. To date, although there is some putative evidence that MBPs may contribute to enhanced leadership, little is known about how MBPs "in the 
real world" are experienced by leaders and what might help leaders to engage with MBPs. This study aims to examine the experience of leaders who participated in an MBP, with a secondary aim of how they applied mindfulness to their leadership role.

\section{Method}

\section{Participants}

To meet inclusion criteria, participants had to (1) be UK-based leaders in a global manufacturing organization (with at least two years' experience in a management role) and (2) have attended an MBP as part of their leadership development in the 2 years prior to the study. Ten leaders aged between 34 and 57 years old participated in this study. Further participant demographic details are shown in Table 1; pseudonyms were allocated to protect their anonymity.

\section{Procedure}

The Ethics Committee of Bangor University granted ethical approval. Potential participants were identified by a leadership development specialist within the organization $(N=54)$. Each received an email introducing the project, and then those interested in participating were invited to contact the first author for more information. Ten participants responded, and all agreed to participate. None of the participants had any prior experience of mindfulness before attending the MBP. Dates for the semi-structured interviews were mutually agreed and took place in work-based locations convenient for each participant.

Participants were reminded at the start of the semi-structured interview that the focus of the interview was their experience of attending the second MBP module of their leadership program. Examples of questions asked included: "What is your understanding of what is required of someone in a leadership role in this organization?"; "What was your expectation of how participating in the MBP would support you in your leadership role?"; "How would you describe your overall experience of the MBP?"; "What did you learn from this?"; and "From what you now know about mindfulness, how might it support you in your leadership role?" On completion of the study all participants received a summary of the findings and a link to access the full study.

\section{The Mindfulness-Based Programme}

The MBP was the second of four modules included within a leadership programme, called the Experienced Manager Program (EMP). The MBP was delivered away from the office environment via a three-day residential retreat, in a rural location. The program was taught by one of four experienced and internationally known mindfulness teachers. Participants were encouraged to disconnect from their day to day work and just be there for the experience for the duration of the programme.

The curriculum was a combination of mindfulness practice with psychoeducation. All the core practices from the 8-week MBSR course (MBSR curriculum guide, 2017) were taught and made relevant to leadership development, such as the body scan and understanding stress reactivity. Mindfulness teachers encouraged participants to continue 


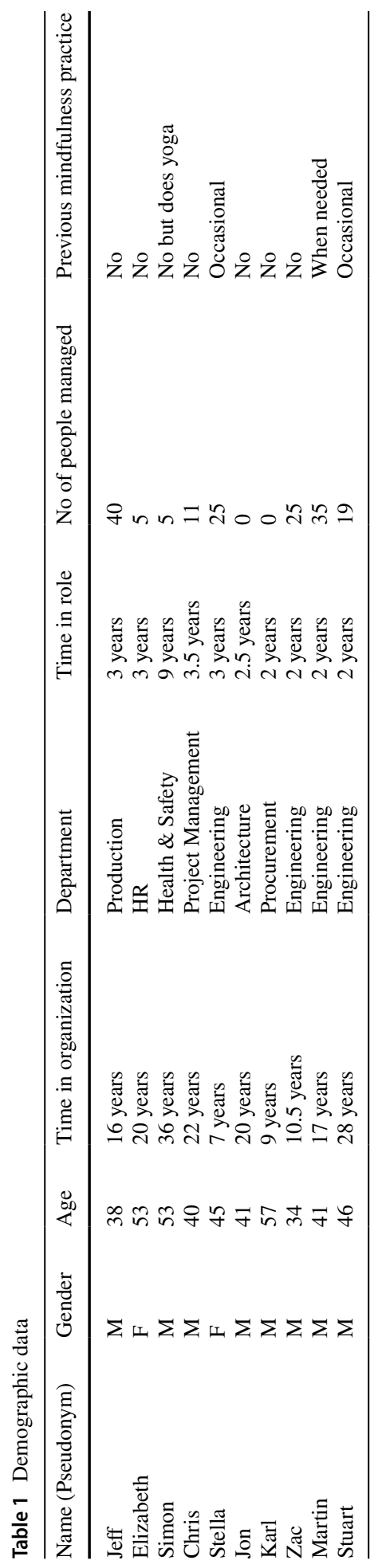


daily mindfulness practices at home for at least 4 weeks after the program. The content of the 3-day MBP is shown in Table 2.

\section{Data Analysis}

Thematic analysis was used to analyse the data, as it is particularly suitable for exploring a subject about which little is known (Braun \& Clarke, 2006; Clarke et al., 2015; Larkin, 2015). The interview data was analysed using the six-step process outlined by Braun and Clarke (2006). These steps were as follows: familiarization with the data, generating initial codes, searching for themes, reviewing themes, defining and naming themes, and producing the report.

The familiarization stage involved the first author listening to all the recordings twice before beginning transcription. This stage continued during transcription and with reading and re-reading of the data once the transcription was complete. The next stage of the thematic analysis involved coding the data by writing notes against all the content on each script that appeared to be pertinent. This process was repeated twice for each transcript to ensure that all possible themes were generated. For the third stage "Searching for themes" the researcher used a process of mind mapping, which involved clustering and linking themes identified. Next, the themes were checked back against the whole data set (stage four). The researcher then continued to stage five (consolidating and naming themes) and stage six, the final write-up. To enhance the reliability of the data analysis, the first author kept notes of observations and reflections throughout the analysis in a reflexive diary to protect against bias as suggested by Braun and Clarke (2006). The first author is a trainer for the company, and she is also a mindfulness teacher (but did not teach these participants), so a potential for a positive interpretation of the data was identified. Care was taken to ensure that all participant data (especially negative or indifferent views) were represented fully in the analysis. Furthermore, themes were triangulated with discussion and input from the second author during stages three to six of the process, and with the third author at stage six of the analysis.

\section{Results}

We identified three superordinate themes, each of which contained two to four subordinate themes. These are represented in Table 3.

\section{Key}

\section{(...) Piece of text omitted}

[ ] Word or piece of text replaced or inserted

\section{The Participant Journey: Mixed Experiences of the MBP}

This superordinate theme explores the direct experience of participants during the MBP. It focuses on the content of the curriculum, program delivery, and the environment. All participants reported that the MBP was unfamiliar and challenging at first. However, most 


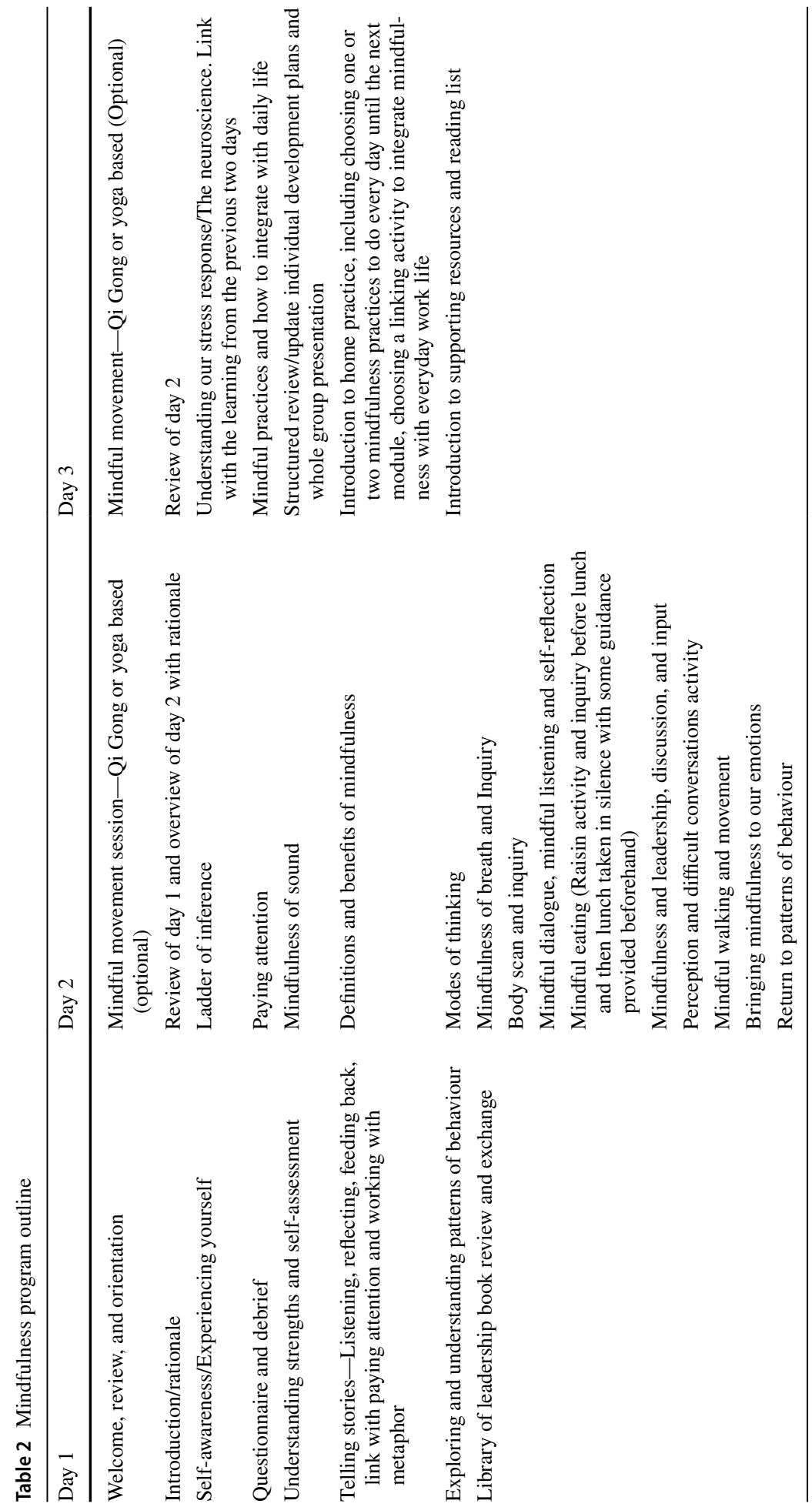


Table 3 Superordinate and subordinate themes

\begin{tabular}{lc}
\hline Superordinate themes & Subordinate themes \\
\hline 1. The participant journey: Mixed experiences of the MBP? & 1.1 Out of their comfort zone \\
& 1.2 Make room for theory \\
& 1.3 It's all about the teacher \\
& 1.4 The Environment \\
2. A clash of cultures: The challenge of integrating mindfulness into the & 2.1 Questioning the approach \\
workplace & 2.2 Stigma \\
3. Impact on leadership & 3.1 A foundation for leader- \\
& ship \\
& 3.2 Mindfulness enhanced \\
& emotional intelligence \\
& 3.3 Significance for health \\
& 3.4 There is no time
\end{tabular}

participants simultaneously recognized the value of the MBP and said they gained skills that they were able to use both in and out of the workplace.

\section{Out of their Comfort Zone}

Most participants knew very little about mindfulness at the start of the module. They described arriving for the 3-day intensive MBP feeling very unprepared: "We are not prepared at all. You just turn up and it happens, and sometimes that can be a bit of culture shock" (Jon).

Several participants commented on the experiential approach used to deliver the MBP, which focused on exploring here and now experience, Stella described it as "Very different to anything else that I have ever experienced" and said that she had found it "exposing" and "tough." Similarly, Jon stated: "Some of the activities we participated in weren't natural for me - I felt a bit uncomfortable." Both participants were initially challenged by the immersive and experiential approach.

Jon went on to describe how by the end of the MBP, both himself and most people in his cohort could see how they might benefit from mindfulness. He mentioned that he-and many of his co-workers-were naturally sceptical but once they understood the potential in the approach they were "won over." The biggest factor in this for Jon seemed to be the opportunity to try out something new and have a direct experience of the effects of mindfulness practice and he said, "It does bring a different perspective."

It is important to acknowledge the views of one participant, Jeff, who did not enjoy the MBP. He held strong opinions about separating what people choose to do personally from their working life. His view was that if people were interested in mindfulness, it was up to individuals to use it, rather than as a mandatory part of a leadership program. As such he felt a sense of coercion to both attend and to develop a practice:

It could have been a little less forced and kind of rammed down your throat... when it feels forced, I don't know that it works - certainly not for my type... It's trying to connect it, having a framework, so maybe it's about how to provide that framework without making it feel, like its rammed down or forced.

Jeff did not discern any connection between mindfulness and leadership development, and at the start of the interview repeatedly used strong language (forced/rammed) to 
describe his experience of felt coercion. Later in the interview he spoke of how he may have responded better to the MBPs had it been delivered in shorter sessions over a longer period of time.

\section{Make Room for Theory}

Several participants reported that it was helpful to learn about the psychoeducational aspects of the MBP, such as the stress reaction cycle and neuropsychology, as they could easily relate to these concepts. Stella liked "The different theoretical aspects of how your brain processes and reacts to different sorts of information." Others also appreciated the unpacking of their stress reactivity.

It was an explanation of why, when something happens - your mind turns to jelly, and you can't do something...So, when something happens and you freeze, it's because you are getting a kind of overload...I now understand... why didn't I do that straight away and why couldn't I think my way out of that? (Karl).

These psychoeducational aspects of the curriculum were appreciated as particularly helpful, possibly because participants were more familiar and comfortable with theory-based training which they could immediately relate to their own experiences of high stress.

\section{It Is All About the Teacher}

For many of the participants, the relational qualities and presence of the MBP teacher was a significant part of their training experience and learning. "I often think that what makes a good course is the person taking it rather than the actual content" (Zac).

Zac described his MBP teacher as "inspirational" and noted that "He was a kind of role model for me, you know, I have often reflected on some of the things that he said." Some felt that the course being led by an experienced mindfulness practitioner who had "walked the walk" with years of their own mindfulness practice was vital.

To have it delivered by someone who practices it and has got the benefits of it...is really compelling and really believable... If you had, you know a communication trainer then it wouldn't have been the same - because you know, he was delivering his life (Simon).

A couple of participants however, reported quite the opposite, and felt that their MBP teacher was inauthentic. It was not possible to discern from the data whether these participants were talking about the same teacher.

To be (...) leading the way he was, there was something not quite right for me, there was something inauthentic about it...I don't like inauthentic people... if someone is saying one message but they don't really believe it. Normally, it doesn't work, if they are acting a certain way and it's not their normal way of behaving (Jeff).

\section{The Environment}

The MBP was delivered in a rural retreat setting. Participants were encouraged to switch off their phones and laptops for the duration of the course. Although challenging at first, most considered this a real support to their learning. Stella described the venue as "calming" 
and Stuart said that "the atmosphere helped with slowing down a bit." This was further expanded on by Stella and Karl:

A wonderful setting, perfect for the module, we went on a mindfulness walk around the grounds...we had a part of one of our evening meals in silence, so yes, the environment was very supportive of the material (Karl).

Hearing the birds, no traffic and the warmth...things like that really resonated with me and I think it really helps you to remember the learning -that complete immersion in the concept of it. (Stella).

Two participants also spoke of the benefits of the MBP not being held in their workplace: "People could disconnect and weren't generally working and checking emails and things like that during break-times" (Zac) and "Had it been at work, it would have been very negative, I reckon. I don't think that we would have got the same from it" (Jon). The experience of these participants indicates that being free of everyday workplace distractions supported their learning and allowed them to connect with their present moment experience.

\section{A Clash of Cultures: The Challenge of Integrating Mindfulness into the Workplace}

At first mindfulness and the workplace seemed to be separate worlds to participants, and initially, they struggled to link the two, but with time, most spoke of the helpfulness of using mindfulness in the workplace, whereas a couple did not feel that mindfulness was of relevance to their leadership role. This theme raises questions about the compatibility of the world of business and mindfulness-based approaches, and how mindfulness might best be presented to those in leadership positions.

\section{Questioning the Approach}

All participants wanted to be able to apply their learning from the MBP module to improve their leadership skills. However, many felt that the link between mindfulness and improved leadership was not always made explicit by the trainer:

The trainer (...) did not, have a very clear link to the company or even any kind of corporate training... his experience was linked to mindfulness but not, completely in our kind of context and I question, whether, he was fully clear...on what, the goals were in terms of the context of our business (Martin).

Here, Martin indicates that in his view the trainer, although experienced in mindfulness was not aligned with the business-related needs of the group. Similarly:

I was thinking well hang on a minute, you haven't got all that much experience, you certainly haven't got, that experience in work...so please don't pretend you do have all the answers... So it just felt like the wrong fit (Jeff).

If you had real world, managers and leaders who had found it had benefitted them, and could tell you their stories, to bridge that gap (...) if there was this middle ground of someone who came from our world and had dipped into their world and had found some usefulness (Stuart).

Some participants remained uncertain of the value of mindfulness and the relevance of it to leadership: "I would still be asking some questions - around well why then, what is it going to do for me? How is it going to help me be a better leader?" (Elizabeth). The main motivation for participants attending the program was to improve their leadership 
skills, and the connection between doing the mindfulness exercises and leadership was not obvious to some either during the course, or after it. Some of the participants felt that they would have benefitted from being taught by a mindfulness teacher who had corporate experience, or who made the links between mindfulness and leadership clear.

\section{Stigma}

Several participants spoke of a stigma they associated with mindfulness which made some hesitant about the approach. Some thought that mindfulness was for people prone to mental health issues, for example: "I guess I don't see myself as someone who is at high risk of succumbing to things like anxiety or mental health challenges" (Zac). Thus, at an identity level, Zac did not regard himself as someone who would benefit from mindfulness. Martin also saw the impact of this stigma on other participants in his cohort: "Some people were less open to mindfulness because it's a very sort of, soft skills or its one of these things... where you can be uncomfortable connecting with it."

Other participants saw the stigma as operating at a more systemic level. Simon said "There is a stigma to someone meditating or doing yoga - that would be seen as weak...I think there is a big stigma attached to it all. I think that's one of the things that...we need to address." Similarly, Jeff described the culture in his workplace as "old school." He suggested this would create a significant barrier to introducing MBPs given the perceived stigma of mindfulness in his workplace:

It would have to be done in a very effective way, I think for people to listen, you know there's all the cynicism and silliness around it and you know it's a topic that can easily be dismissed as sort of hippyish and that type of thing (Jeff).

\section{Impact on Leadership}

Most participants made it clear that the questions and challenges raised about the compatibility of mindfulness approaches and their workplace culture were not insurmountable. Indeed, the majority spoke of how, after the MBP, they had incorporated mindfulness in their leadership role and/or in their personal lives. Half had made some positive changes, a couple thought that they might use mindfulness in the future or if they needed to, and a few remained sceptical of mindfulness as an approach for leadership development. This superordinate theme illustrates how participants related mindfulness to their leadership role, and how some implemented their personal mindfulness practice in the workplace.

\section{A Foundation for Leadership}

Several participants spoke of the importance of self-awareness, trust, and knowing when to pause as being essential qualities of leadership which-they discovered -could be supported by a mindfulness practice. Karl said the MBP was particularly useful for people seeking progression in their careers. He placed considerable emphasis on the connection between self-awareness, mindfulness, and the organization's leadership model: "That introduction of that kind of awareness is very important. Linked back to the leadership model- you know, certain elements of being yourself." He described how he witnessed mindfulness approaches applied in later leadership training, and it was at this point-after the MBP - that he realized the potential usefulness of mindfulness in the workplace. He recalled a moment when participants were preparing to take part in a role-play activity: 
The leader sensed the tension in the room. And you know, she calmed that... she said right let's just stop for a moment, let's just practice some mindfulness, - just for a minute. Bring back something that you learnt in [the MBP] - and that worked for me and I know that it worked for other people as well.

Others spoke of how they had made connections between leadership and mindfulness, from developing higher awareness of the self: "Trusting your own strengths and developing self-awareness. That's the kind of thing that I think that it helped with" (Stuart) and also to being able to take pauses in the day when needed: "I certainly learnt about the ability to stop...in any situation to, if necessary, just stop, be aware" (Chris).

Martin felt that in the past, he had not always acted as a leader, but following the MBP he feels more confident in doing so: "Feeling it's ok to display some leadership behaviours got reinforced through that course and through the mindfulness element in particular."

Many participants also spoke about their enhanced capacity to decentre following the MBP. This is the process of stepping back from our own thoughts and viewing them objectively. Decentring led to a wider awareness, which helped them to be aware of multiple perspectives. Jon, for example, stated:

The intent is to look at yourself, look at your surroundings be more mindful of the whole situation, so look at it from a holistic point of view, not necessarily from your immediate task or your immediate objective which people tend to focus on in work.

Zac also commented on how he developed awareness of the transience of moods and the importance of being more aware of his mood states during the working day (another aspect of decentring);

In my role, sometimes I can go back-to-back meetings, all day and I think making that link, that perhaps what happened in a previous session, may have a knock-on effect to the next session, in terms of my state of mind going into that session.

Several participants stated how mindfulness meditation had helped their emotional reactivity and enabled them to step back and observe their feeling states without being fused with them. This then allowed them to recognize how an emotion might directly impact on their behaviour, which helped them to choose how to respond to the situation, rather than have an emotional reaction. Karl stated:

I have recognized that in certain emotional states, I might not be in the best state to carry out certain things... and therefore - ok - shall I park it? Shall I talk to somebody? Shall I do something different? But I won't do an emotional response.

Karl spoke of how decentring allowed him to pause rather than immediately reacting to an emotion, which allowed him to approach situations at work once he had calmed down. "I certainly learned about the ability to stop (...) in any situation, to if necessary, just stop, be aware, just take those few breaths." He then gave a recent example of this:

It actually happened to me this morning, I got an email and my initial reaction was ummm - [so], no, I am not even going to draft a reply right now. I recognize the way I am feeling...is probably not the right frame of mind...I am too emotional and also, the way I thinking right now, I may not gather all the facts that I need... so I am not going to respond to it right now, I may not even respond to it at all.

Chris also said that he now understood that: "You can take a kind of a step back, how you can calm yourself, how you can think things through before reacting." The majority of participants explicitly stated that reduced stress leads to better leadership, and many found that the increases in self-awareness, decentering skills, and awareness of emotional reactivity all enabled them to helpfully work with their levels of stress at work. Indeed, at the end of the interview, Zac said that of all the modules attended, the MBP is the one that he "took the most away from." He explained: 
It's probably a bit kind of airy fairy - would have been my initial reaction but...out of the four modules, it's the one that I have made the most use of and it has probably changed my kind of thinking or improved my behaviours the most ( $\mathrm{Zac}$ ).

\section{Mindfulness Enhanced Emotional Intelligence}

Many participants felt that mindfulness practice could underpin the development of their emotional intelligence as leaders. Some spoke of how they learned to prioritize self-care more, and others shifted from a focus on achieving goals to creating a positive process by which those goals are reached and the interpersonal aspects of this process. Jon said; "The module taught me that you need, to think about yourself, give yourself time, not just think about yourself but about others around you." This was echoed by Martin who stated, "It's helped to focus on setting up the environment and the way things are working with the team, rather than directly focusing on the topic somehow."

For some participants, the MBP allowed them to reflect on their habitual patterns of responding and how these could directly impact on others they worked with. Stella spoke of her habit of withdrawing when challenged in meetings and described how mindfulness did not stop the initial reaction of wanting to withdraw, but allowed her instead to notice this, and in that small gap, she could choose to respond in a different way:

... which might make you want to shrivel up and disappear. And, it's important that you don't do that, so if you can give yourself the time to respond back and say well no, look at it from this perspective.

So, in the context of sometimes challenging meetings, enhanced awareness has enabled Stella to actively contribute her ideas. This was supported by Zac who noted the direct positive impact that mindfulness had on him both in and outside of work:

I get angry, significantly less, following that module, basically, whilst I might get frustrated, I can focus on not getting angry, whereas before, I had a tendency to get angry from time to time in work and outside of work.

He noted that some meetings continued to be challenging, yet he found that the application of mindfulness can bring about a positive change in "hostile" exchanges: "So just to like create some space... so rather than to engage with it or to start some conflict, just to kind of slow down if I feel any frustration or tension." Another benefit of having the skill to pause and notice, was noted by Stella who reported that she now takes "time to notice what's around me" and notes how this generates a "different perspective." All these contributions indicate that utilizing mindfulness as an approach when interacting with others has a wide range of applications. These include pausing in meetings and being more able to regulate emotional responses, which are helpful to the leader's stress levels and also likely to facilitate interaction with colleagues.

\section{Significance for Health}

Participants recognized the importance of good physical and mental health for people in leadership roles, and experiencing the MBP led to an enhanced focus on their own wellbeing for some. In describing life for many leaders, Simon said: 
Their health gets worse and worse, they don't look after themselves, they don't take lunchbreaks, they work long hours and they don't give themselves any time to breathe... So, I see mindfulness as massively important... it could help prevent burn-out.

The relationship between how stress could have a negative impact on leadership was also noted. Elizabeth said:

I can see a link between me being a better leader... if I am less stressed and calmer in approach...If a team member comes to me and asks me for something, then of course, I want to give them all my attention. Without, perhaps, conveying to them how completely stressed I am.

Stella also spoke of the importance of focusing on well-being and recognized that some of her own everyday habits could, if left unchecked, contribute to burnout: "The focus on well-being is absolutely key. It's so easy to spend my lunchtime eating my sandwich and focusing on something that needs to be done. That's become a habit." She felt the whole workforce, not just leaders, could accrue health benefits from self-care as part of a mindfulness practice.

Others commented on how the application of particular mindfulness practices helped them to unwind from the working day and sleep. For example, Jon said: "When I am giving people difficult messages... I can't switch off. I take that home and I think about it at night." He then reflected that: "This is where the mindfulness has helped - trying to get to sleep - that deep breathing." He continued saying that he would use mindfulness practice on an 'as and when' basis: "I wouldn't do it every day or once a week. I would do it when I am in that situation thinking, right I can't sleep or whatever."

These comments demonstrate the variety of ways in which participants applied mindfulness practices. Some said they now sometimes allocated time for self-care, which helped them focus at work by supporting their well-being: "Before the module, I had absolutely no appreciation that you need to give yourself that time and "be"... and post module, consciously I am doing that now" (Jon).

\section{There is no Time}

Many participants spoke of their experience of working in a highly pressurized work environment. The description below seems to capture a felt frantic-ness at work that was also expressed by other participants:

If you are not in a meeting or not progressing some activity or another over the phone or via email or by talking to someone face-to-face, then you are not productive. And you have to be productive, apart from when you are having your break, all the time, every single minute of the day because otherwise that's wasting time (Elizabeth).

In line with the perception that leaders always need to be engaged in "activity," most participants reported, time constraints as a barrier to mindfulness practice. Stuart said, that at work "I don't have a break from the start of the day to the end of the day." Others talked of highly pressured home lives too, and how fitting in mindfulness practice was difficult: "Whether I do that in my personal life - not really because it's always go, go, go, work, rest, or play - you know, there is always something to do" (Jon).

Elizabeth reported personal barriers around prioritizing her health and creating time for herself, saying: "It's me finding some time for me... It doesn't happen." Some participants felt they couldn't prioritise mindfulness practice because as Elizabeth noted, it seemed to be something personal rather than directly addressing the 'to do' list 
or need to achieve something tangible. Stella also described a desire to create time for herself to help her mental health, whilst feeling unable to do so:

I think I could get a lot more from it than I do. I am such a busy person... where do you get that time bubble...for you to just de-stress, before you yell at somebody? So, I think there is a real opportunity there but what I need to do is find how to build that in (Stella).

A couple of participants reported that they wanted to develop a personal mindfulness practice, but found it challenging to implement after the course: "I think that to some extent, I suffered from the classic - I have been away on a training course and then slammed back into real life" (Karl). Similarly, Martin said:

My intention... was to take time out every day for a few minutes breathing type meditation, and I haven't really. If I am being completely honest - I think I did it in a halfhearted way a couple of times not long after the course. This was partly because I was struggling. I needed to adjust my lifestyle a bit, there were just too many things going on.

The time pressures of working life, and not having support after the MBP course, led to most participants being unable to establish a regular, formal meditation practice:

It fell by the wayside...the suggestion is, do them for thirty days and it's not until you do them regularly that you see the benefits. So, in that sense, I am a little bit disappointed in myself. Looking back and saying if it really takes that long to see a benefit then I really should have pushed it through, to see would I gain the benefit or not. I probably gave up too quickly on some of them (Chris).

Participants spoke of how a single three-day MBP with no follow-up support was not enough to embed an ongoing regular mindfulness practice. Some participants would have found follow-up sessions helpful and motivating. Indeed, one of the participants (Karl), suggested that if they were told that the mindfulness practices would be actively worked with in later leadership trainings then "There is no doubt, I would have done it." Another, (Jeff) said he thought that rather than including this approach in just one of the modules of the LDP, it would have been better supported by spreading it across all four leadership modules, "Bringing it into each day." In addition, Elizabeth said she would have benefitted had the mindfulness teacher spoken more about how to establish a regular mindfulness practice "If we had had some opportunity to share our concerns about where am I going to find this time to apply it? That wasn't factored in and maybe more of a group discussion. Some way of just sharing." Most participants could see the potential benefits of practicing mindfulness. However, although some participants spoke about using mindfulness when they felt the need, or applying mindfulness in specific situations, none reported sustaining a regular, formal mindfulness practice after the MBP.

\section{Discussion}

This study examined participant experiences of a three-day MBP attended as a part of a leadership development programme, and the impact of this on their leadership role. Participants reported a wide range of experiences and outcomes, and the majority spoke of direct benefits to themselves and their leadership role. Two, however, did not report any benefits, and were left unsure of how to apply the MBP in the workplace, raising important questions about implementation, and the role and presentation of mindfulness in leadership development programs in the community.

Most participants spoke of how the MBP facilitated self-awareness, self-confidence, emotional intelligence, and ability to manage stress, which in turn enhanced their 
leadership skills. Many authors note the importance of these skills gained by participants as foundational for authenticity in leadership (Hawkins, 2018; Hughes et al., 2009; Northouse, 2013; Rishel, 2015). It is therefore encouraging that some participants reported increased self-awareness and emotional intelligence as a result of the MBP.

Before discussing the implications of these findings, it is important to acknowledge some limitations of this study; results of qualitative studies are not intended to be generalizable, but instead to offer an insight into the lived experience of participants. Having said this, however, we can assume that if an idea or theme is repeated by a number of interviewees, we might be able to putatively generalize that others in similar contexts may have experienced something similar (Braun \& Clarke, 2013). Another limitation is that this was a self-selected group, of mostly men. However, there were a range of experiences captured in this sample, from those who found the MBP useful to those that remained sceptical of the relevance of mindfulness to leadership. Future studies may also benefit from a member-check with participants to ensure that the results are representative of the experiences expressed in the interviews.

This study also only included leaders from one organization, and whose MBP was delivered in the format of a unique 3-day retreat. For the future it will be important to seek the views of participants in a range of other organizations, and who have learned about mindfulness in different formats. This might help develop an optimum format for delivering MBPs to leaders. One final limitation is that this study only included the views of the people who had participated in the training. In future research it would be helpful to interview colleagues and team members of leaders who have been on mindfulness training to examine whether they observed differences in leadership after the leader has participated on an MBP course.

Some participants reported that at times it was challenging to learn about mindfulness, noting a felt tension between the culture of the workplace and mindfulness. For some, mindfulness carried a stigma of being "hippy" or for people with psychological difficulties. Most participants were able to assimilate this over time and saw how mindfulness was relevant for leadership, although it is important to note that two did not. Mindfulness-based approaches are focused on process and experiential learning, whereas workplaces tend be goal or outcome orientated. This makes it—understandably-difficult for some to see the connection between engaging in a body scan mediation for example and being a better leader. The challenges of this "clash of cultures" is not unique to leadership and is part of the adaptation of mindfulness-based interventions for different settings (Crane, 2016). Some participants felt this clash would have been eased had the mindfulness teacher had a corporate background and was able to demonstrate how mindfulness is helpful to leaders. This point is supported by Ruderman and Clerkin (2017), who noted that language and the experiential learning approach of mindfulness may be a barrier to some operating in a business environment. Similarly, participants also wanted more evidence on the benefits of mindfulness regarding leadership development to be included in the MBP, resonating with the findings of Lewis and Ebbeck (2014). Addressing these barriers may support strategies to help increase the adoption of MBPs into leadership programs (NIH model Stage V: Onken et al., 2014). It may also be important to build on some of the enablers mentioned by other participants in the current study such as an initial introduction to mindfulness in a quiet environment away from the workplace and having an MBP teacher who leaders can relate to. These are identified by various authors writing about teaching and learning in a wide variety of related contexts (Goleman \& Senge, 2014; Palmer, 2007; McCown et al., 2010). Furthermore, Goldman Schuyler et al. (2017) state that a "radical shift" is necessary if people are to apply mindfulness in the workplace. They continue by noting both the 
level of commitment and preparedness to face ambiguity, required at an individual level in engaging with MBPs, acknowledging that "Not everyone wishes to do this."

Mindfulness-based programs have been shown to reduce levels of stress among general populations (Baer, 2003; Kabat-Zinn, 2003; Kuyken et al., 2008, 2015). Participants in this study reported this too, and furthermore pointed to the mechanisms of how this may have worked. They described how mindfulness helped them to recognize their stress and, respond to it (e.g., by pausing or taking a few breaths), which then allowed them to see their experience from a wider perspective, and thus less likely to fall into usual patterns of reactivity. This last step is a mechanism of mindfulness known as decentering (Segal et al., 2013) or re-perceiving (Shapiro et al., 2006), and the findings tentatively suggest that decentering may be a key mechanism by which leaders may be able to approach challenges while acknowledging the impact that stress may be having. This in turn, may lead to enhanced self-care and perhaps lower stress and reactivity, and is therefore worthy of future research.

Half the participants reported that they applied mindfulness skills to their lives and in the workplace, others said that they would if they ever needed it, and two remained sceptical of mindfulness as a helpful tool for leaders. Examples of changes made included: using mindfulness of the breath to support sleep; recognizing when it may not be wise to make a decision; recognizing and implementing choices in inter-personal communication; managing anger, and taking steps to improve well-being at work. Similarly, the leaders in Reitz et al. (2016) reported a range of benefits which they attributed to their experience of an MBP. In terms of leadership, the majority of participants in the current study specifically made a link between feeling less stressed and being a better leader. They said that they considered that being less stressed improved their capacity to pay attention, make decisions, and develop emotional intelligence. These are all considered to be essential competencies for leaders (Hougaard et al., 2016; Hyland et al., 2015; Reitz et al., 2016).

Two participants did not feel the MBP supported them to be a better leader. Of note is that these participants did not like the mindfulness-based activities or consider them relevant to their professional roles. One felt that the MBP should be optional (rather than a core part of the LDP) and did not perceive any connection between mindfulness and leadership development. These comments are important to note for the future integration and sustainability of LDP's incorporating mindfulness-based approaches. To support engagement, information on the rationale for including MBP's in LDPs needs to be made explicit to leaders, alongside evidence of the benefits of the approach, and some examples of how other leaders have applied mindfulness at work.

Participants who intended to establish a mindfulness practice when they returned to work after the MBP found they struggled to find the time to do so. This resonates with the findings reported by Reitz et al. (2016), who found that leaders, having completed an MBP, also reported the challenge of finding both time and a supportive environment to practice. While many found the retreat aspect of the MBP beneficial for immersion in and exploration of mindfulness ideas and practice, some participants struggled to conflate these with their roles and daily reality. This may point to the need to ensure aspects of MBPs for leaders are experienced in a workplace setting too.

Senge et al. (2015), acknowledged the commitment and discipline required of leaders to become more effective and noted that this is "hard work." They stressed the value of the support of others undertaking similar journeys. Therefore, if the potential benefits of MBPs for leaders are to be fully realized, organizations may need to consider providing post-program support. This could include setting aside a time and space for leaders to practice mindfulness together, for example a weekly lunchtime mindfulness practice open to all employees. It may also be that successful implementation of a mindfulness-based 
approach to leadership in organisations will require the commitment of multiple stakeholders in different contexts as described by Goleman and Senghe (2014). So, an area for future research is to determine what further workplace adaptions could support leaders to maintain a mindfulness practice.

Authors' Contributions DD: Designed the study, collected the data, and led the article. KN: Contributed to the design of the study, the data analysis, writing, and editing, GG: Contributed to the data analysis, original draft preparation, writing and editing of the study. All authors read and approved the final manuscript.

\section{Compliance with Ethical Standards}

Conflict of Interest The authors declare that they have no conflict of interest.

Ethical Approval All procedures performed in studies involving human participants were in accordance with the ethical standards of the institutional and/or national research committee and with the 1964 Helsinki declaration and its later amendments or comparable ethical standards.

Informed Consent Informed consent was obtained from all individual participants included in the study.

Open Access This article is licensed under a Creative Commons Attribution 4.0 International License, which permits use, sharing, adaptation, distribution and reproduction in any medium or format, as long as you give appropriate credit to the original author(s) and the source, provide a link to the Creative Commons licence, and indicate if changes were made. The images or other third party material in this article are included in the article's Creative Commons licence, unless indicated otherwise in a credit line to the material. If material is not included in the article's Creative Commons licence and your intended use is not permitted by statutory regulation or exceeds the permitted use, you will need to obtain permission directly from the copyright holder. To view a copy of this licence, visit http://creativecommons.org/licenses/by/4.0/.

\section{References}

All Party Parliamentary Group. (2015). Mindful nation - UK. Retrieved from http://www.themindfulnessinitiative. org.uk/.

Baer, R. A. (2003). Mindfulness training as a clinical intervention: conceptual and empirical review. Clinical Psychology: Science and Practice, 10, 125-143. https://doi.org/10.1093/clipsy.bpg015.

Braun, V., \& Clarke, V. (2006). Using thematic analysis in psychology. Qualitative Research in Psychology, 3(2), 77-101. https://doi.org/10.1191/1478088706qp063oa.

Braun, V., \& Clarke, V. (2013). Successful qualitative research: A practical guide for beginners. London: Sage.

Brendel, W., \& Bennett, C. (2016). Learning to embody leadership through mindfulness and somatics practice. Advances in Developing Human Resources, 18(3), 409-425. https://doi.org/10.1177/1523422316646068.

Brendel, W., Hankerson, S., Bryun, S., \& Cunningham, B. (2016). Cultivating leadership dharma measuring the impact of regular mindfulness practice on creativity, resilience, tolerance for ambiguity, anxiety and stress. Journal of Management and Development, 35, 1056-1078. https://doi.org/10.1108/ JMD-09-2015-0127.

Center for mindfulness in medicine, health care, and society (CFM) University of Massachusetts Medical school. (2017). Mindfulness-based stress reduction (MBSR) authorized curriculum guide.

Chaskalson, M. (2011). The mindful workplace: developing resilient individuals and resonant organizations with MBSR. Chichester: Wiley-Blackwell.

Clarke, V., Braun, V., \& Hayfield, N. (2015). Thematic analysis. In J. A. Smith (Ed.), Qualitative psychology (pp. 222-248). London: Sage.

Crane, R. S. (2016). Implementing mindfulness in the mainstream: making the path by walking it. Mindfulness, 8, 585-594. https://doi.org/10.1007/s12671-016-0632-7.

Dimidjian, S., \& Segal, Z. V. (2015). Prospects for a clinical science of mindfulness-based intervention. American Psychologist, 70(7), 593.

Ehrlich, J. (2017). Mindful leadership: focusing leaders and organisations. Organisational Dynamics, 46, 233-243. https://doi.org/10.1016/j.orgdyn.2017.05.002. 
Financial Services Authority Board Report. (2011). The failure of the Royal Bank of Scotland. Retrieved from https://www.fca.org.uk/publication/corporate/fsa-rbs.pdf.

Germer, C. K. (2005). Teaching mindfulness in therapy. Mindfulness and Psychotherapy, 1(2), 113-129.

Gill, R. (2011). Theory and practice of leadership. Thousand Oaks, CA: Sage.

Goldman Schuyler, K., Skjei, S., Sanzgiri, J., \& Koskela, V. (2017). "Moments of waking up". A doorway to mindfulness and presence. Journal of Management Inquiry. https://doi.org/10.1177/1056492616665171.

Goleman, D., \& Senge, P. (2014). The triple focus. A new approach to education. LLC, Florence: More than Sound.

Hawkins, P. (2018). Tomorrow's leaders and the necessary revolution in today's leadership development. Retrieved from https://s3-eu-west-1.amazonaws.com/assets.henley.ac.uk/legacyUploads/pdf/exec-ed/ Research_Report.

Hougaard, R., Carter, J., \& Coutts, G. (2016). Mindful leadership—achieving results by managing the mind. Leader to Leader, 79, 49-56. https://doi.org/10.1002/It1.20218.

Hughes, R. L., Ginnett, R. C., \& Curphy, G. J. (2009). Leadership enhancing the lessons of experience (6th ed.). Singapore: McGrawHill.

Hyland, P. K., Lee, R. A., \& Mills, M. J. (2015). Mindfulness at work: a new approach to improving organisational and individual performance. Industrial and Organisational Psychology, 8(4), 576-602. https://doi.org/10.1017/iop.2015.41.

Kabat-Zinn, J. (2003). Mindfulness-based interventions in context: past, present, and future. Clinical Psychology: Science and Practice, 10, 144-156. https://doi.org/10.1093/clipsy/bpg016.

Kabat-Zinn, J. (2013). Full catastrophe living: how to cope with stress, pain and illness using mindfulness meditation (Revised and (updated). London: Piaktus.

Khoury, B., Sharma, M., Rush, S. E., \& Fournier, C. (2015). Mindfulness-based stress reduction for healthy individuals: a meta-analysis. Journal of psychosomatic research, 78(6), 519-528.

King, E., \& Haar, M. J. (2017). Mindfulness \& job performance: a study of Australian leaders. Asia Pacific Journal of Human Resources, 55, 298-319. https://doi.org/10.1111/1744-7941.12143.

Kuyken, W., Byford, S., Taylor, R. S., Watkins, E., Holden, E., White, K., \& Teasdale, J. D. (2008). Mindfulness-based cognitive therapy to prevent relapse in recurrent depression. Journal of Consulting and Clinical Psychology, 76, 966-978. https://doi.org/10.1037/a0013786.

Kuyken, W., Hayes, R., Barrett, B., Byng, R., Dalgleish, T., Kessler, D., \& Byford, S. (2015). Effectiveness and cost-effectiveness of mindfulness-based anti-depressant treatment in the prevention of depressive relapse or recurrence (PREVENT): a randomised control trial. The Lancet, 386, 63-73.

Larkin, M. (2015). Choosing your approach. In J. A. Smith (Ed.), Qualitative psychology. London: Sage, $250-256$.

Lewis, A. B., \& Ebbeck, V. (2014). Mindful and self-compassionate leadership development: preliminary discussions with wildland fire managers. Journal of Forestry, 230-236. J. For. 112(2):230-236. https:// doi.org/10.5849/jof.12-107.

McCown, D., Reibel, D., \& Micozzi, M. S. (2010). Teaching mindfulness: a practical guide for clinicians and educators. New York: Springer.

Northouse, P. G. (2013). Leadership: theory and practice (6th ed.). Thousand Oaks, CA: Sage.

Onken, L. S., Carroll, K. M., Shoham, V., Cuthbert, B. N., \& Riddle, M. (2014). Reenvisioning clinical science: unifying the discipline to improve the public health. Clinical Psychological Science, 2(1), 22-34.

Palmer, P. J. (2007). The courage to teach: exploring the inner landscape of a teacher's life. San Francisco, CA: Jossey-Bass.

Reb, J., Narayanan, J., \& Chaturvedi, S. (2014). Leading Mindfully: two studies on the influence of supervisor trait mindfulness on employee well-being and performance. Mindfulness, 5, 36-45. https://doi. org/10.1007/s12671-012-0144-z.

Reitz, M., Chaskalson, M., Olivier, S., \& Waller, L. (2016). The mindful leader- developing the capacity for resilience and collaboration in complex times through mindfulness practice. Hertfordshire, UK: Hult Research.

Rishel, C. J. (2015). The role of resilience and mindful leadership in oncology nursing. Oncology Nursing Forum, 42(2), 198-199. https://doi.org/10.1188/15.ONF.198-199.

Ruderman, M. N., \& Clerkin, C. (2017). Using mindfulness to improve high potential development. Retrieved from https://www.cambridge.org/core.

Segal, Z. V., Williams, J. M. G., \& Teasdale, J. D. (2013). Mindfulness-based cognitive therapy for depression ( $2^{\text {nd }}$ ed.) New York: The Guilford Press.

Senge, P., Hamilton, H., \& Kania, J. (2015). The dawn of system leadership. Winter: Stanford Social Innovation Review, 27-33. 
Shapiro, S. L., Carlson, L. E., Astin, J. A., \& Freedman, B. (2006). Mechanisms of mindfulness. Journal of Clinical Psychology, 62(3), 373-386. https://doi.org/10.1002/jclp.

Wasylkiw, L., Holton, J., Azar, R., \& Cook, W. (2015). The impact of mindfulness on leadership effectiveness in a health care setting: a pilot study. Journal of Health Organisation and Management, 29(893-911), 6. https://doi.org/10.1108/JHOM-06-2014-0099.

Publisher's Note Springer Nature remains neutral with regard to jurisdictional claims in published maps and institutional affiliations. 